\title{
Whole-Genome Sequencing and Annotation of the Yeast Clavispora santaluciae Reveals Important Insights about Its Adaptation to the Vineyard Environment
}

\author{
Ricardo Franco-Duarte ${ }^{1,2, *(\mathbb{D})}$, Neža Čadež ${ }^{3}(\mathbb{D})$, Teresa Rito ${ }^{1,2}\left(\mathbb{D}\right.$, João Drumonde-Neves ${ }^{4}$, \\ Yazmid Reyes Dominguez ${ }^{5}$, Célia Pais ${ }^{1,2} \mathbb{D}^{\mathbb{D}}$, Maria João Sousa ${ }^{1,2} \mathbb{D}^{\mathbb{D}}$ and Pedro Soares ${ }^{1,2}$
}

check for

updates

Citation: Franco-Duarte, R.; Čadež,

N.; Rito, T.; Drumonde-Neves, J.;

Dominguez, Y.R.; Pais, C.; Sousa,

M.J.; Soares, P. Whole-Genome

Sequencing and Annotation of the

Yeast Clavispora santaluciae Reveals

Important Insights about Its

Adaptation to the Vineyard

Environment. J. Fungi 2022, 8, 52.

https://doi.org/10.3390/jof8010052

Academic Editor: Brian Monk

Received: 6 December 202

Accepted: 4 January 2022

Published: 5 January 2022

Publisher's Note: MDPI stays neutral with regard to jurisdictional claims in published maps and institutional affiliations.

Copyright: (C) 2022 by the authors. Licensee MDPI, Basel, Switzerland. This article is an open access article distributed under the terms and conditions of the Creative Commons Attribution (CC BY) license (https:// creativecommons.org/licenses/by/ $4.0 /)$
1 CBMA, Centre of Molecular and Environmental Biology, Department of Biology, University of Minho, 4710-057 Braga, Portugal; teresarito@bio.uminho.pt (T.R.); cpais@bio.uminho.pt (C.P.); mjsousa@bio.uminho.pt (M.J.S.); pedrosoares@bio.uminho.pt (P.S.)

2 Institute of Science and Innovation for Bio-Sustainability (IB-S), University of Minho, 4710-057 Braga, Portugal

3 Department of Food Science and Technology, Biotechnical Faculty, University of Ljubljana, 101, 1000 Ljubljana, Slovenia; neza.cadez@bf.uni-lj.si

4 IITAA-Institute of Agricultural and Environmental Research and Technology, University of Azores, 9700-042 Angra do Heroísmo, Portugal; drumondeneves@gmail.com

5 Laimburg Research Centre, Laimburg 6, 39052 Vadena, Italy; Yazmid.Reyes-Dominguez@laimburg.it

* Correspondence: ricardofilipeduarte@bio.uminho.pt or ricardofrancoduarte@gmail.com

\begin{abstract}
Clavispora santaluciae was recently described as a novel non-Saccharomyces yeast species, isolated from grapes of Azores vineyards, a Portuguese archipelago with particular environmental conditions, and from Italian grapes infected with Drosophila suzukii. In the present work, the genome of five Clavispora santaluciae strains was sequenced, assembled, and annotated for the first time, using robust pipelines, and a combination of both long- and short-read sequencing platforms. Genome comparisons revealed specific differences between strains of Clavispora santaluciae reflecting their isolation in two separate ecological niches-Azorean and Italian vineyards-as well as mechanisms of adaptation to the intricate and arduous environmental features of the geographical location from which they were isolated. In particular, relevant differences were detected in the number of coding genes (shared and unique) and transposable elements, the amount and diversity of noncoding RNAs, and the enzymatic potential of each strain through the analysis of their CAZyome. A comparative study was also conducted between the Clavispora santaluciae genome and those of the remaining species of the Metschnikowiaceae family. Our phylogenetic and genomic analysis, comprising 126 yeast strains (alignment of 2362 common proteins) allowed the establishment of a robust phylogram of Metschnikowiaceae and detailed incongruencies to be clarified in the future.
\end{abstract}

Keywords: genomics; phylogenomics; functional gene analysis; Metschnikowiaceae; Azores; wine yeasts; biotechnology; adaptation

\section{Introduction}

In our previous surveys on the yeast diversity of Azorean vineyards, in 2009 and 2010 [1-3], we described a new yeast species Clavispora santaluciae [4], isolated from grapes. It was characterized on the basis of the sequences of the internal transcribed spacer (ITS) region (ITS1-5.8S-ITS2), the sequences of the D1/D2 domain of the large subunit (LSU) rRNA gene, and particular physiological characteristics. That study also described this species as being isolated from grapes infected with Drosophila suzukii in Italy. This showed identical D1/D2 sequences and very similar ITS regions (five nucleotide substitutions) to the Azorean strains. The new species was obtained from particular viticultural environments, typical of the Azores archipelago, which result from the interaction between specific climatic conditions, autochthonous grapevine cultivars, and local viticultural practices. Phenotypic characterization of this new species revealed some interesting features that 
positioned it apart from the closely related ones, such as the inability to grow at temperatures above $35^{\circ} \mathrm{C}$, production of acetic acid, and the capacity to assimilate starch. The full biotechnological potential of this new species remains to be explored, as does the understanding of the genomic features associated with the adaptation to its environment. The occurrence of biotechnologically important features associated with species of this clade is not uncommon. (Candida) intermedia, a xylose-utilizing species of the Metschnikowiaceae family, belonging to the Clavispora clade, displays a high-capacity xylose transport system $[5,6]$. Due to these characteristics, this species has been categorized as an attractive species to produce ethanol from lignocellulosic biomass [7].

Our previous study was one of the few to report the presence of yeast species belonging to the Clavispora clade in vineyards, even though some rare reports have already associated these yeasts with winemaking, as detailed below. We highlighted the rarity of these occurrences, in a review of the association of non-Saccharomyces yeasts with viticulture and winemaking [8]. In that study, we systematized 80 years of the literature describing nonSaccharomyces yeast species isolated from grapes and/or grape musts and compiled a list of 293 species. Only two species belonging to the Clavispora clade were identified-Clavispora fructus and Clavispora lusitaniae. Even though there is no strong association between Clavispora species and wine, some reports have already described the possible advantages of these yeasts in the production of wines with alternative sensory characteristics, albeit with some associated disadvantages, such as the presence of an abnormally high concentration of acetaldehyde [9]. In Azorean vineyards, no other yeast of this genus was found within the 2910 isolates identified in our previous work [1,2], but a single species of this family (Metschnikowia pulcherrima) was found in four different islands in two consecutive sampling years. The use of this species in wine biotechnology was recently reviewed [10], with the authors concluding that its versatility lies in its ability to ferment must in combination with other yeast species (mainly to circumvent its low fermentative power), as well as modulating the synthesis of secondary fermentation metabolites to improve and diversify the sensory profile of the wine.

The phylogram obtained in our previous study [4], based on concatenated sequences of the D1/D2 domain of the LSU rRNA gene, and of the ITS region, placed Clavispora santaluciae strains near closely the related species Clavispora fructus, (Candida) asparagi, (Candida) vitiphila, (Candida) phyllophila, (Candida) carvajalis, and Clavispora lusitaniae. The description of this new species was considered as an important add-on in understanding the phylogenetic relationships within the Clavispora clade and clarifying their biodiversity and ecology. Previously, several yeasts that now belong to the Clavispora genus, were placed in the anamorphic genus Candida, due to their inability to form sexual spores [11]. With recent phylogenetic analysis based on DNA sequences, in combination with physiological evidence, the relationships between the species of Candida and the genus Clavispora began to be clarified, leading to a new classification of this group by Daniel et al. in 2014 [11], with 40 species of Candida assigned to the genus Clavispora, based on sequences of the LSU D1/D2 domain, ITS region and four coding genes (ACT1, TEF1, MCM7, and RPB2). In 2018, Kurtzman et al. [12] described four new species of Metschnikowia and proposed to transfer seven additional Candida species to both Metschnikowia and Clavispora genera. Kurtzman et al. concluded that the taxonomy of the Clavispora clade could only be clarified by whole-genome comparisons, which still need to be performed. Regarding the Clavispora genus, only two species have been genome sequenced and annotated: Clavispora lusitaniae (11.9-12.1 Mb, 8 chromosomes, genome accession ASM167369v2 ), and Clavispora fructus (11.4 Mb, NCBI genome accession ASM370779v1). In 2016, a phylogeny of the Metschnikowiaceae family was presented by Lachance et al. [13], combining draft genomes of 55 strains and identifying 3016 orthologues, 1061 of which exist in all the analyzed strains. Even though the Clavispora lusitaniae genome was used as a query to compare between all the genomes, no species belonging to the Clavispora genus were considered, with the analysis focused on only the Metschnikowia genus. More recently, Shen et al. [14] attempted to reconstruct the phylogeny of 300 budding yeast species, focusing mainly on 
the diversity of Saccharomycotina. In that work, whole genomes were used to partially describe the phylogeny of Metschnikowiaceae clade considering only 22 Metschnikowia species. This analysis used only the type strains of each species, which lack intra-species diversity, and the genome annotation was not directed to the analysis of this clade. Thus, a deep, broad, and focused analysis using robustly annotated genomes of the phylogenetic relations between Clavispora species (including also the recently assigned Candida species) and the sister genus Metschnikowia is lacking. With this in mind, the objective of the present work was to sequence, assemble and annotate whole genomes of the different Clavispora santaluciae strains, using a combination of both long- and short-read sequencing platforms, in order to obtain high-quality sequences for comparative genomics. We used the assembled genomes to further elucidate the molecular mechanisms underlying the adaptation of this species to the particular environmental characteristics from which it was isolated. In particular, the main goal was to unravel the genomic features that can explain the phenotypic characteristics previously observed within isolates of Clavispora santaluciae, as well as to predict their biotechnological potential. In addition, all species from the Metschnikowiaceae family whose complete genome was publicly available were considered and combined in phylogenetic and genomic analysis, to help clarify the phylogenetic placement of Clavispora yeasts within this large group of important non-Saccharomyces yeasts. We plan to use our results to clarify the positioning of (Candida) species in relation to the sister genus Metschnikowia and reconstruct Metschnikowiaceae family phylogeny using complete genomes.

\section{Materials and Methods}

\subsection{Cell Culture, Sample Collection, and DNA Extraction}

The type strain of Clavispora santaluciae $\left(\mathrm{A} 1.18^{\mathrm{T}}=\mathrm{CBS} 16465^{\mathrm{T}}\right)$, together with the three strains isolated from grapes of Azorean vineyards (A1.5, A1.7, and A1.19), and one additional strain isolated from grapes infected with Drosophila suzukii in Italy (LB-NB-3.3) [4], were grown in YPD broth (yeast extract, $1 \% w / v$; peptone, $1 \% w / v$; glucose $2 \% w / v$ ), in $50 \mathrm{~mL}$ conical flasks, for $48 \mathrm{~h}$ at $28{ }^{\circ} \mathrm{C}, 220 \mathrm{rpm}$. Genomic DNA was isolated according to the protocol published by Schwartz and Sherlock [15], with a few adaptations for the isolation of DNA from non-Saccharomyces yeasts. After washing in $0.9 \mathrm{M}$ sorbitol solution, the cells were incubated by adding $20 \mu \mathrm{L}$ of Lyticase $(30 \mathrm{mg} / \mathrm{mL}$, Sigma-Aldrich, St. Louis, MO, USA) to the cells. The incubation time was at least $4 \mathrm{~h}$ at $37^{\circ} \mathrm{C}$. Following phenol/chloroform (Millipore, Burlington, MA, USA) extraction, the DNA was precipitated using $40 \mu \mathrm{L}$ of $3 \mathrm{M}$ sodium acetate ( $\mathrm{pH}$ 5.5) and $1 \mathrm{~mL}$ of absolute ethanol and resuspended in $200 \mu \mathrm{L}$ of TE buffer.

\subsection{Genome Sequencing and Assembly}

The genomes of all the Clavispora santaluciae strains were sequenced by using a combination of the long-and short-read sequencing technologies of PacBio and Illumina, respectively. After DNA extraction, library preparation and PacBio/Illumina sequencing were performed at Novogene facilities (Novogene Company LTD, Cambridge, United Kingdom). Low-quality reads and adapters were removed by Novogene, and sequencing quality was accessed using FastQC software (http:/ / www.bioinformatics.bbsrc.ac.uk/projects/fastqc/; accessed on 1 August 2021). The sequencing data are available at NCBI BioProject ID PRJNA784374.

Long-reads obtained from PacBio sequencing were de novo assembled using Canu v.1.9 [16] with default parameters. Illumina paired-end reads were then used to improve assembly quality, using Masurca software v.4.0.5, in particular the Polca package $[17,18]$. Finally, RagTag software v.2.1.0 [19] was used to assemble all the scaffolds into longer reads, using chromosome information from the closely related species Clavispora lusitaniae and (Candida) intermedia. Genome assembly quality metrics, available in Table 1, were computed using QUAST v.5.0.2 [20]. 
Table 1. Genome assembly statistics of Clavispora santaluciae strains.

\begin{tabular}{|c|c|c|c|c|c|c|}
\hline & & A1.18 ${ }^{\mathrm{T}}$ & A1.5 & A1.7 & A1.19 & LB-NB-3.3 \\
\hline \multirow{7}{*}{ 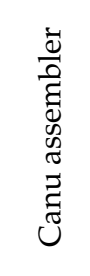 } & Assembly length (bp) & $11,088,431$ & $11,018,248$ & $10,921,443$ & $10,861,576$ & $11,019,028$ \\
\hline & Number of scaffolds & 43 & 13 & 46 & 86 & 30 \\
\hline & N50 (bp) & 315,943 & 802,369 & 355,153 & $1,329,122$ & 494,470 \\
\hline & L50 & 7 & 3 & 6 & 14 & 7 \\
\hline & Number of N's per $100 \mathrm{~Kb}$ & 0 & 0 & 0 & 0 & 0 \\
\hline & Number of scaffolds $>5000 \mathrm{bp}$ & 29 & 11 & 28 & 53 & 23 \\
\hline & Total length $>5000 \mathrm{bp}$ & $10,780,215$ & $10,974,595$ & $10,557,056$ & $10,118,395$ & $10,856,688$ \\
\hline \multirow{9}{*}{ 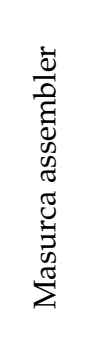 } & Substitution errors revised & 42 & 8 & 141 & 428 & 70 \\
\hline & Insertion/Deletion errors revised & 1686 & 536 & 2825 & 6144 & 1607 \\
\hline & Assembly length (bp) & $11,089,145$ & $11,018,616$ & $10,922,446$ & $10,863,639$ & $11,019,715$ \\
\hline & Number of scaffolds & 43 & 13 & 46 & 86 & 30 \\
\hline & N50 (bp) & 532,329 & $1,048,728$ & 654,799 & 218,696 & 650,701 \\
\hline & L50 & 7 & 3 & 6 & 14 & 7 \\
\hline & Number of N's per $100 \mathrm{~Kb}$ & 0 & 0 & 0 & 0 & 0 \\
\hline & Number of scaffolds $>5000 \mathrm{bp}$ & 29 & 11 & 28 & 53 & 23 \\
\hline & Total length $>5000 \mathrm{bp}$ & $10,780,920$ & $10,974,959$ & $10,558,055$ & $10,120,373$ & $10,857,358$ \\
\hline \multirow{7}{*}{ 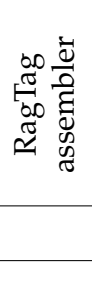 } & Assembly length (bp) & $11,092,545$ & $11,019,016$ & $10,925,846$ & $10,870,339$ & $11,021,815$ \\
\hline & Number of scaffolds/chromosomes & 9 & 9 & 12 & 19 & 9 \\
\hline & Number of N's per $100 \mathrm{~Kb}$ & 3065 & 3.63 & 31.12 & 61.64 & 19.05 \\
\hline & Number of scaffolds $>5000 \mathrm{bp}$ & 4 & 8 & 4 & 3 & 7 \\
\hline & Total length $>5000 \mathrm{bp}$ & $11,025,073$ & $11,000,234$ & $10,766,609$ & $10,606,285$ & $10,966,127$ \\
\hline & Ploidy & haploid & haploid & haploid & haploid & haploid \\
\hline & GC content $(\%)$ & 49.66 & 49.70 & 49.73 & 49.66 & 49.76 \\
\hline
\end{tabular}

To determine ploidy, we used nQuire software [21] to align sequencing reads to the type strain genome assembled after RagTag and determine base frequency distributions between frequencies 20 and 80. Assessment of each genomes' completeness was performed using Benchmarking Universal Single-Copy Orthologs (BUSCO) software v.5.2.2 [22]. Average nucleotide identity (ANI) was calculated using the OrthoANIu web tool [23] in pairwise mode, to compare the nucleotide content of genomes.

\subsection{Genome Annotation}

Annotation of Clavispora santaluciae genome assemblies was performed using AUGUSTUS software v.3.4.0 [24,25], considering 11 different pre-trained models, chosen as belonging to the Ascomycota phyla Saccharomyces cerevisiae S288c, Candida albicans, Meyerozyma (Candida) guilliermondii, Candida tropicalis, Debaryomyces hansenii, Eremothecium gossypii, Kluyveromyces lactis, Lodderomyces elongisporus, Scheffersomyces (Pichia) stipitis, Schizosaccharomyces pombe, and Yarrowia lipolytica. Results were manually reviewed to select the annotation with the higher number of predicted coding genes, which was obtained using Lodderomyces elongisporus as the pre-trained model, for all the Clavispora santaluciae strains. The potential coding regions (nucleotide sequences) reported by AUGUSTUS were extracted from the complete genomes to FASTA files.

CMsearch [26] and StructRNAfinder [27] were used for screening the presence of non-coding RNA (ncRNA). The Rfam database [28] was employed for ncRNA searching, using an e-value of 0.01 .

Functional genomic annotation was performed with eggNOG-mapper v.2 [29] by considering proteins predicted by AUGUSTUS and choosing only orthologs that were inferred from the experimental evidence. The results were described considering clusters of orthologous groups (COGs) with their associated functional categories [30], and also considering the Kyoto Encyclopedia of Genes and Genomes (KEGG) pathways, in particular the KEGG Orthology (KO) descriptors [31,32]. Gene function predictions were also accomplished by assessing the Carbohydrate-Active EnZymes (CAZymes) database [33]. 
Final genome annotations for all Clavispora santaluciae strains are available in Supplementary Data S1.

\subsection{Homology Analysis, Comparative Genomics, and Phylogenomics}

To compare the genome of Clavispora santaluciae type strain A1.18 with that of the remaining strains, dot plots were produced using the Re-Dot-Able tool (https:/ / www. bioinformatics.babraham.ac.uk/projects/redotable/). Inter-species differences between members of the family Metschnikowiaceae were evaluated by downloading all the complete genomes publicly available at NCBI (121 strains belonging to 48 different species). When more than one strain was available for a certain species, all strains were considered. The exception was (Candida) auris for which only the representative genome was used since the hundreds of strains with genome sequence available would have increased redundancy.

KEGG Mapper was used as a collection of KEGG mapping tools for linking genes and proteins to metabolic pathways [32,34]. In particular, $\mathrm{KO}$ gene annotations, obtained from eggnog-mapper, were used to assess pathway completeness using KEGG MapperReconstruct web tool (www.genome.jp/kegg/mapper/reconstruct.html). Results were applied in the construction of a heatmap using Microsoft Excel ${ }^{\circledR}$.

A database was prepared by considering all 126 complete genomes (121 strains of Metschnikowiaceae family plus the 5 Clavispora santaluciae isolates). To avoid inconsistency, the 121 Metschnikowiaceae genomes were annotated using Augustus with the same pre-trained model as was applied for the annotation of the Clavispora santaluciae genomes. BLASTP analysis was performed using the full proteome of the Clavispora santaluciae type strain $\mathrm{A} 1.18^{\mathrm{T}}$ as a query against the total database. An $E$-value cutoff of $10^{-6}$ was used to exclude false results, and a pipeline adapted from [35] was used to perform comparative genomics between all isolates. The BLASTP results were filtered where representative proteins were detected in the other 121 isolates. Each set of probable homologous proteins (containing the query and the respective results) were multiple aligned using the MAFFT algorithm in FasParser (https:/ / github.com/Sun-Yanbo/FasParser) [36]. All proteins from a given organism were concatenated using the alignment results to obtain the core conserved aligned proteome containing mostly essential genes not related to specific biological traits of each species. This alignment was then used for phylogenetic reconstruction by considering the maximum likelihood in IQ-TREE (www.iqtree.org) [37], with the JTT model of amino acid evolution and gamma-distributed rates (four rates) with 500 bootstrap replicates. Two outgroups were considered: Lipomyces lipofer and Cyberlindnera jadinii. FigTree v.1.4.4 (http:/ / tree.bio.ed.ac.uk/software/figtree/) was used to visualize and edit the tree. The second round of BLASTP analysis, using the proteomes of the five Clavispora santaluciae strains as queries, allowed building Venn diagrams to schematize the number of genes common between the five genomes using the average results between all pairs or between groups of three, four, or in all five strains.

\section{Results and Discussion}

\subsection{Sequencing, De Novo Assembly, and Annotation of Clavispora Santaluciae Genome}

Genome sequencing of Clavispora santaluciae strains $\mathrm{A} 1.18^{\mathrm{T}}, \mathrm{A} 1.5, \mathrm{~A} 1.7, \mathrm{~A} 1.19$, and LB-NB-3.3 was performed using a combination of long- and short-read sequencing platforms. Between 27,903 and 44,977 reads were obtained with long-read sequencing, with a maximum read length of 110,418 base pairs (bp). Short-read sequencing was used to refine long-read sequencing results. An average value of $3 \times 10^{6}$ paired-end reads, with $250 \mathrm{bp}$ each, was obtained for each strain. The first round of assembly was performed using Canu and Masurca assemblers (sequencing statistics are presented in Table 1), and then RagTag software assembled the scaffolds into putative chromosomes. By using three assemblers we were able to assemble long and short-read sequences into full chromosomes for three of the strains, including the type strain $\mathrm{A} 1.18^{\mathrm{T}}$ and strains A1.5 and LB-NB-3.3. The remaining two strains, possibly due to lower sequencing depth, were only assembled into large scaffolds. The attained haploid genome size $(10.8 \mathrm{Mb}$ to $11.1 \mathrm{Mb})$ was comparable with 
the previously published genomes of Clavispora yeasts, in particular with the 11.9-12.1 Mb of Clavispora lusitaniae (8 chromosomes) [38,39], or with the $11.4 \mathrm{Mb}$ of Clavispora fructus (NCBI genome accession ASM370779v1).

The high-quality-assembled genomes allowed the prediction of between 6015 and 6092 protein-coding genes for the five Clavispora lusitaniae strains using AUGUSTUS software (Table 2, Supplementary Data S1). These values are among the highest reported for yeasts of the Clavispora clade, and are comparable only to the annotation of one ((Candida) intermedia strain YCC 4715), for which 6082 coding genes were predicted [40], but corresponding to a greater genome length of $13.08 \mathrm{Mb}$.

Table 2. Clavispora santaluciae genome annotation statistics.

\begin{tabular}{|c|c|c|c|c|c|}
\hline & A1.18 & A1.5 & A1.7 & A1.19 & LB-NB-3.3 \\
\hline \multicolumn{6}{|l|}{ Protein coding genes } \\
\hline Total number & 6092 & 6034 & 6067 & 6015 & 6038 \\
\hline Range of protein lengths (aa) & $66-4974$ & $63-4974$ & $57-4974$ & $60-4974$ & $66-5293$ \\
\hline Average protein length (aa) & 557.6 & 556.6 & 550.9 & 543.5 & 518.3 \\
\hline \multicolumn{6}{|l|}{ Non-coding RNAs } \\
\hline microRNAs (miRNAs) & 32 & 32 & 33 & 31 & 21 \\
\hline small RNAs (sRNA) & 20 & 21 & 22 & 20 & 23 \\
\hline nuclear RNAs (snRNA) & 7 & 7 & 6 & 7 & 7 \\
\hline nucleolar RNAs (snoRNA) & 93 & 91 & 99 & 94 & 98 \\
\hline long noncoding RNAs (lncRNA) & 8 & 8 & 9 & 8 & 12 \\
\hline ribosomal RNAs (rRNA) & 96 & 63 & 42 & 69 & 124 \\
\hline transfer RNAs (tRNA) & 276 & 259 & 279 & 299 & 248 \\
\hline Other & 29 & 32 & 32 & 35 & 32 \\
\hline \multicolumn{6}{|l|}{ BUSCO Orthologs } \\
\hline \multicolumn{6}{|l|}{ Ascomycota odb10 database } \\
\hline Genome Completeness (\%) & 93.5 & 94.4 & 93.4 & 90.7 & 93.6 \\
\hline Complete BUSCOs & 1595 & 1611 & 1594 & 1547 & 1597 \\
\hline Fragmented BUSCOs & 17 & 14 & 18 & 21 & 4 \\
\hline Missing BUSCOs & 94 & 81 & 94 & 138 & 94 \\
\hline \multicolumn{6}{|l|}{ Saccharomycetes odb10 database } \\
\hline Genome Completeness (\%) & 98.0 & 99.1 & 98.0 & 95.1 & 98.2 \\
\hline Complete BUSCOs & 2094 & 2118 & 2094 & 2032 & 2099 \\
\hline Fragmented BUSCOs & 14 & 11 & 12 & 17 & 13 \\
\hline Missing BUSCOs & 29 & 8 & 31 & 88 & 25 \\
\hline \multicolumn{6}{|l|}{ Eggnog-mapper functional annotation } \\
\hline Genes with $\mathrm{KO}$ assigned & $3130(51.4 \%)$ & $3129(51.9 \%)$ & $3125(51.6 \%)$ & $3130(52.0 \%)$ & $3101(51.4 \%)$ \\
\hline Genes with COG assigned & $4180(68.6 \%)$ & $4171(69.1 \%)$ & $4166(68.7 \%)$ & $4119(68.5 \%)$ & $4141(68.6 \%)$ \\
\hline \multicolumn{6}{|l|}{ CAZymes functional annotation } \\
\hline Number of genes annotated & 120 & 121 & 118 & 112 & 117 \\
\hline
\end{tabular}

The unusually high number of predicted proteins in the genome of Clavispora santaluciae was likely not related, in our opinion, to any peculiarity of this yeast's genome but rather to the use of advanced sequencing technologies, together with an improved annotation pipeline. The lowest number of predicted coding sequences was determined for strain A1.19. This could be attributed to lower sequencing depth. This was also the shortest genome of the five, the one with the lowest N50 values (Table 1), and the one with lower BUSCO genome completeness scores, both in Ascomycota and Saccharomycetes databases (Table 2).

The highest number of predicted proteins was described in the annotation of the genome of the type strain $\mathrm{A} 1.18^{\mathrm{T}}$, with 6092 coding sequences (Table 2). The average length of the predicted proteins was slightly lower in LB-NB-3.3, although the largest protein of 5293 amino acids (aa) was annotated in this strain. This large open reading frame encodes the protein midasin (Mdn1), an ATPase of $560 \mathrm{kDa}$ that is essential for cell viability. It was identified in all Clavispora santaluciae strains and reported in other yeasts, such as in the genera Saccharomyces and Schizosaccharomyces, as well as in distant organisms as 
Drosophila and Arabidopsis [41]. The lowest coding sequence annotated (57 aa) corresponds to a hypothetical protein not yet characterized in the Metschnikowiaceae (data not shown) but identified as a mitochondrial ATP synthase $\varepsilon$ chain-domain-containing protein in the Terfezia claveryi mycorrhizal fungus (NCBI accession KAF8454923.1). The fact that we found no proteins below this size, which could correspond to the annotation of false positives, highlights the high annotation quality obtained with the computational pipeline and the sequencing technology applied.

The total number of non-coding RNAs (ncRNA) predicted using structRNAfinder and the Pfam database was similar among the five Clavispora santaluciae strains (Table 2, Supplementary Data S2). There was a high similarity between strains for the majority of the ncRNA annotated, with the exception of ribosomal and transfer RNAs (rRNA and tRNA, respectively), whose quantities showed relevant inter-strain variation not directly correlated with the number of predicted coding sequences or with the genome size. Many sequencing projects ignore the comparison of ncRNA between strains, but by detailing their analysis, it may be possible to understand particular and intricate mechanisms of adaptation to the environment.

\subsection{Comparative Genomics of Clavispora Santalucieae Strains}

To compare structural variations between the genomes of the Clavispora santaluciae strains pairwise, dot plots were obtained (Figure 1A). Results showed a striking pattern of conservation for most strains, with a high degree of macrosynteny mainly between the type strain and strains A1.19 and LB-NB-3.3. On the other hand, strains A1.5 and A1.7 showed some differentiation, in particular by the presence of several deletions in parts of the genome, as represented by translocations ("jumps" in the dot plot) away from the main diagonal. In particular, strain A1.5 seems to have mesosynteny with the type strain, since we can generally observe conservation of the gene content. However, in some parts of the genome, many inversions (blue lines) and translocations were detected. This observation is not concordant with the similarities observed in the ITS and D1/D2 regions [4], which showed that strain A1.5 is most closely related to the type strain.

A

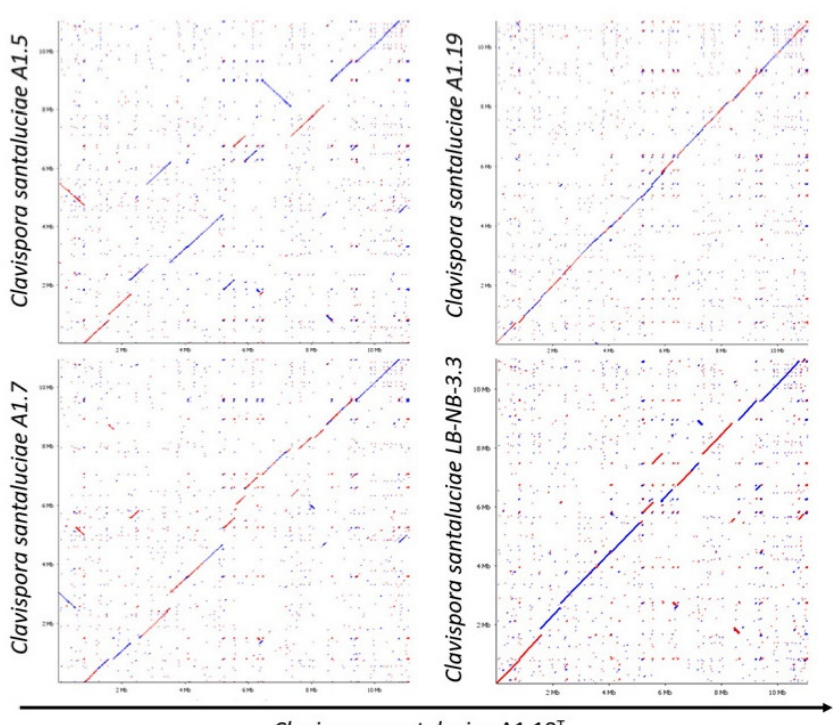

B

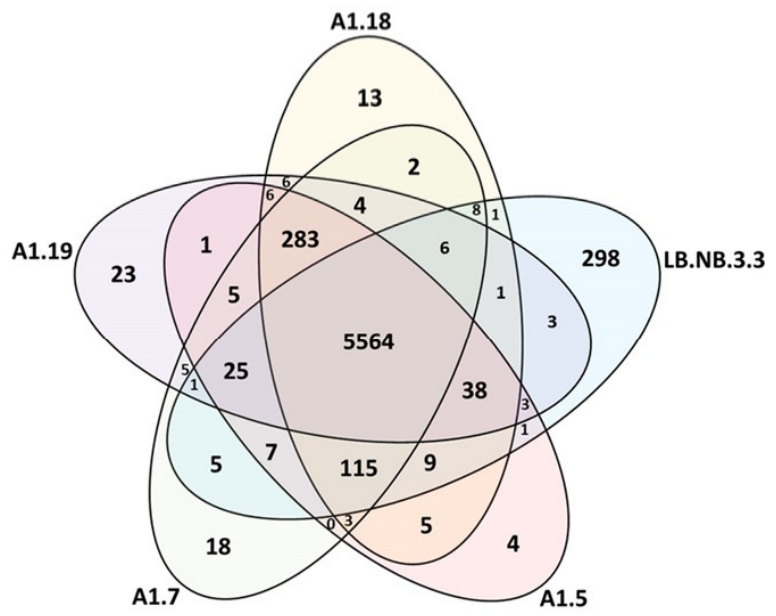

Clavispora santaluciae $\mathrm{A} 1.18^{\top}$

Figure 1. Comparative genomics of Clavispora santaluciae genomes: (A) whole-genome dot-plot comparison between the sequenced strains in pairwise mode. Homologous regions are plotted as dots. Red lines link parallel homologous pairs, and blue lines link anti-parallel pairs; (B) Venn diagram indicating the number of shared coding genes among Clavispora santaluciae strains. 
A total of 5564 coding genes were found to be shared between the five Clavispora santaluciae strains, corresponding to the pangenome of the species (Figure 1B). Strain NBLB-3.3 showed a surprisingly high number of unique genes (298), not shared by any of the other strains, reflecting its adaptation to a different ecological niche, as this strain was isolated from Italian grapes infected with Drosophila suzukii. On the other hand, 283 genes were shared only by the strains isolated from Azorean vineyards, indicating adaptation mechanisms to the intricate and arduous environmental conditions of the geographical location from which they were isolated. Additionally, and of particular note, is the fact that no transposable element was identified in the genome of strain LB-NB-3.3, unlike the other four Azorean strains (Supplementary Data S1). According to our previous work [42] on the characterization of isogenic isolates of wine $S$. cerevisiae yeasts, transposable elements seem to be related to the adaptation of yeasts to the fluctuating environmental conditions found in the harsh environment of the Azores archipelago, and these genetic features are related with important phenotypic characteristics that determine the strains biotechnological potential [43,44].

\subsection{Functional Annotation of Clavispora Santaluciae Proteome}

For this analysis, eggNOG-mapper functionally annotated the predicted open reading frames of Clavispora santaluciae, providing important insights into their biological significance (Table 2, Figure 2). Between 3101 and 3103 genes were assigned to a KO category, corresponding to an average of $51.7 \%$ of all the annotated genes. A total of 4180 genes of Clavispora santaluciae type strain $\mathrm{A} 1.18^{\mathrm{T}}$ (68.6\% of the total genes) were clustered into 24 COGs using eggNOG-mapper (Figure 2A), which were then classified into three main functional categories (Figure 2). This analysis revealed low variation between the five strains which is in accordance with the remaining annotation statistics shown before. Of note is the fact that the number of functionally annotated genes obtained in all strains varied between 68.5 and $69.1 \%$ (Table 2) and is rather low, as indicated by the high number of genes with "unknown function" in panel B of Figure 2 (gray bars; between 20.7 and $20.9 \%$ ). However, these values are lower than those obtained for other species (Figure 2C, and category S in panel D), such as Clavispora lusitaniae, with $24 \%$, (Candida intermedia), with $25 \%$, and Metschnikowia reukaufii, with $24 \%$, or even for Saccharomyces cerevisiae $(22 \%)$ or Torulaspora delbrueckii (22\%), as shown in our previous work [35]. This low number of genes with "unknown function" is a consequence of an improvement in the sequencing and annotation pipelines normally used to annotate yeast genomes.

Functional annotation of Clavispora santaluciae revealed that the highest percentage of annotated genes (Figure 2B) was related to "metabolism" (between 27.3 and 27.7\%), followed by "cellular processes and signaling" (26.4-26.6\%). This result is in agreement with that of other yeasts of Metschnikowiaceae (Figure 2, panels C and D), although this novel yeast species has a higher percentage of genes related to metabolism, which points to a superior biotechnological potential of this species. The importance of this value is even more evident if we compare it with the functional annotations of yeasts from other families, for which usually "information storage and processing" is the most represented category, as is the case of T. delbrueckii and S. cerevisiae, as previously shown [35]. The most abundant COG category in the genome of Clavispora santaluciae $\mathrm{A} 1.18^{\mathrm{T}}$ (panel A) was "translation, ribosomal structure, and biogenesis" (333 genes, representing $8 \%$ of the annotated genes), followed closely by "posttranslational modification, protein turnover, chaperones" (328/7.8\%). The least abundant categories were "extracellular structures", with only two associated genes. 

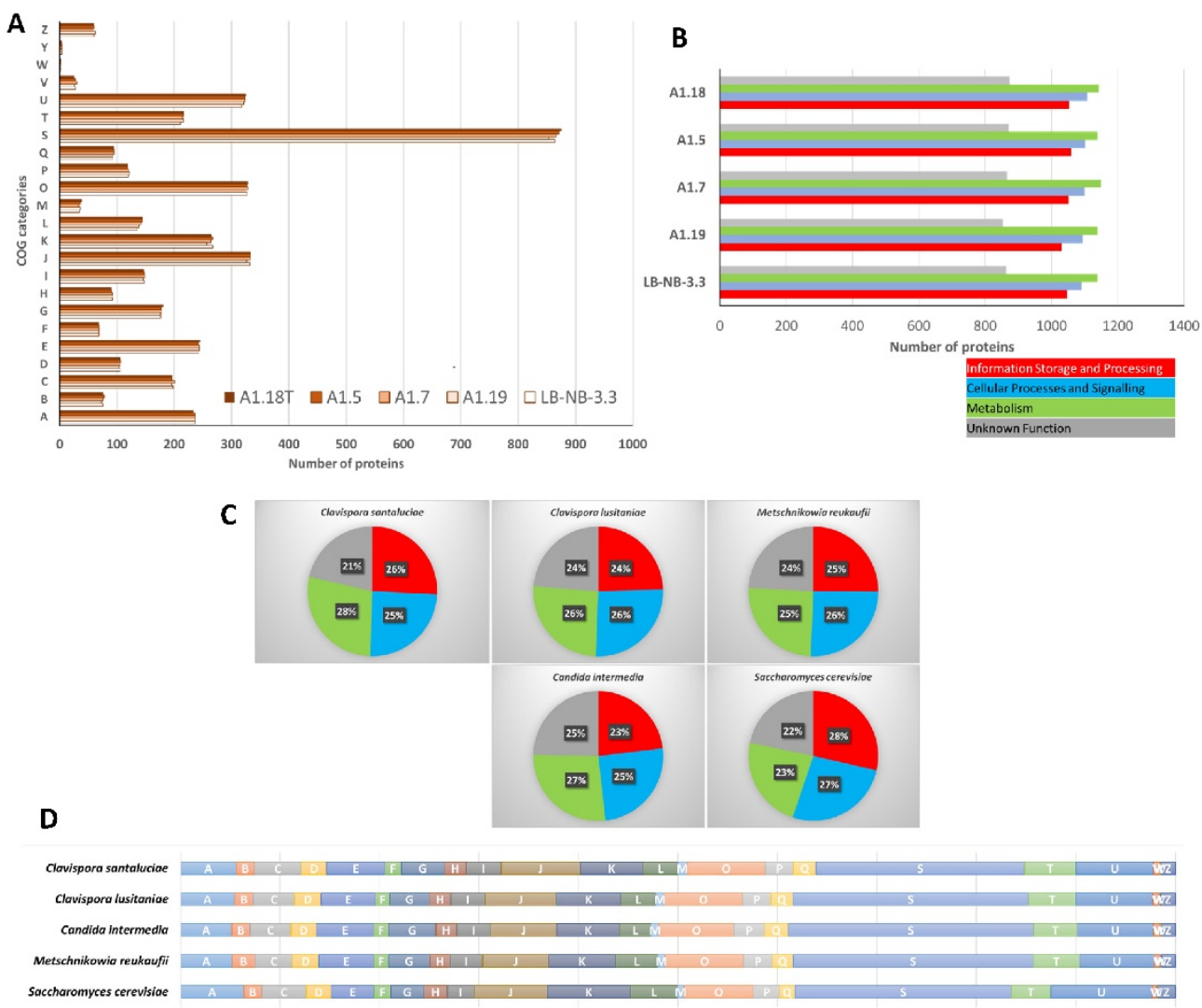

D

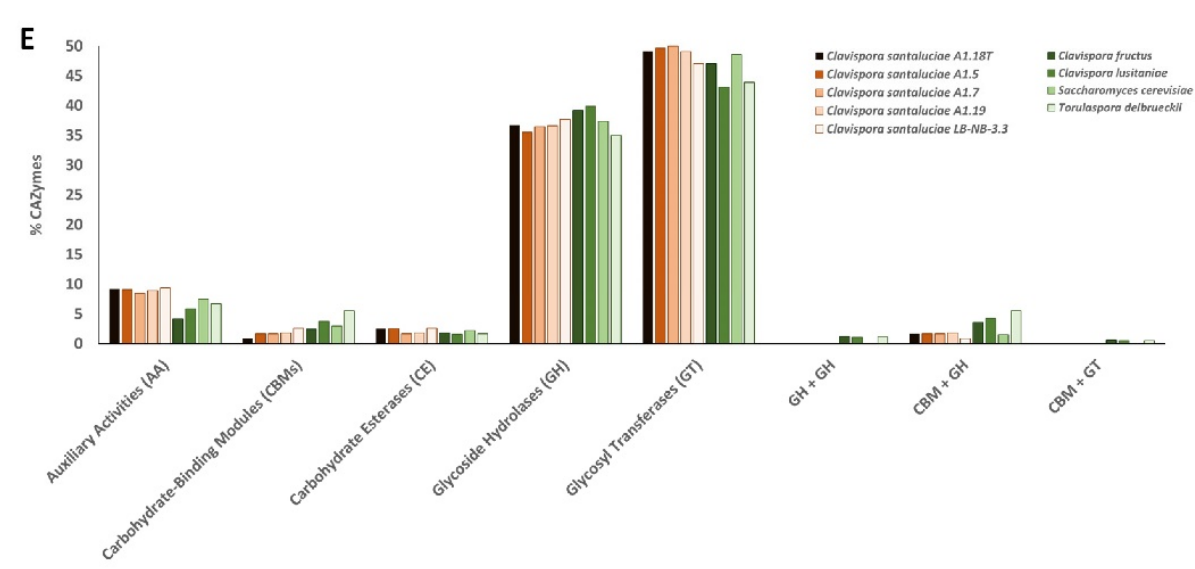

Figure 2. Functional annotation of Clavispora santaluciae genome: (A) proteome classification into 23 functional categories, corresponding to clusters of orthologous groups (COGs): A, RNA processing and modification; B, chromatin structure and dynamics; C, energy production and conversion; D, cell cycle control and mitosis; E, amino acid metabolism and transport; F, nucleotide metabolism and transport; G, carbohydrate metabolism and transport; H, coenzyme metabolism; I, lipid metabolism; $\mathrm{J}$, translation; $\mathrm{K}$, transcription; $\mathrm{L}$, replication and repair; $\mathrm{M}$, cell wall/membrane/envelop biogenesis; $\mathrm{O}$, posttranslational modification, protein turnover, chaperone functions; $\mathrm{P}$, inorganic ion transport and metabolism; $Q$, secondary Structure; $S$, function unknown; T, signal transduction; $U$, intracellular trafficking and secretion; $Y$, nuclear structure; Z, cytoskeleton; (B) classification of the annotated genes into four large functional categories; (C) comparison between the five Clavispora santaluciae strains and other relevant yeast species in proportions of the large functional categories; (D) comparison between relevant yeast species classification of the annotated genes into 23 COG categories; (E) percentage of CAZymes in the five sequenced genomes of Clavispora santaluciae and other relevant yeasts, showing the distribution of predicted proteins into major families. 
Carbohydrate-active enzymes (CAZymes) were identified in the genome of Clavispora santaluciae by searching seven different families: auxiliary activities (AA), proteins containing a carbohydrate-binding module (CBMs), carbohydrate esterases (CE), glycoside hydrolases $(\mathrm{GH})$, glycosyltransferases (GT), polysaccharide lyases (PL) and expansins (EXP), as well as combinations of the categories above (Figure 2E). Analysis of CAZymes revealed between 112 (strain A1.19) and 121 (strain A1.5) putative genes, distributed among five families, as no genes related with PL or EXP were detected. Approximately 1.97\% of the total protein-coding genes in the Clavispora santaluciae genome encode CAZymes, which is in accordance with the reported range of 1 to $3 \%$ described for the generality of prokaryotes and eukaryotes [45].

In Figure 2E small inter-strain differences in Clavispora santaluciae CAZyome are noted, although strains A1.19 and LB-NB-3.3 have a slightly lower number of glycosyltransferases. The two classes with a higher number of annotated genes were glycosyltransferases and glycoside hydrolases. These CAZymes take part in the hydrolysis of glycosidic bonds between two or more carbohydrates or between a carbohydrate and a non-carbohydrate, as in the case of glycosyltransferases, or they assist in the formation of glycosidic bond and biosynthesis of polysaccharides, as in the case of glycosyltransferases. Interestingly, the CAZyome of Clavispora santaluciae reveals a control over complex carbohydrates, either being involved in their assembly (glycosyltransferases) or in their breakdown (glycoside hydrolases). To unravel genomic traits underlying the starch assimilative capacity [4], the presence of the enzymes GH31, GH13, GH57, and GH77 was analyzed since these are associated with an improved capacity to degrade starch [45]. Results (data not shown) revealed that Clavispora santaluciae CAZyome had genes GH31 and GH13, which encode starch degrading enzymes, and their presence could explain the particular capacity of this species. However, closely related species of Clavispora and Metschnikowia branches revealed no differences in the annotation of these glycosyl hydrolases families [46], when compared with the novel species. In fact, despite differences in the total number of CAZymes, no particularly relevant differences were found between Clavispora santaluciae and other species of the same family, regarding important enzymes involved in cellulolytic, hemicellulolytic, and starch degradation (alignments and BLASTP results not shown). As Clavispora santaluciae was isolated solely from grapes, a comparison was performed between its CAZyome and that of the wine yeasts S. cerevisiae and T. delbrueckii (Figure 2E), selected for their involvement in the fermentation process and relevance in the winemaking industry $[44,47]$. Interestingly, all Clavispora santaluciae strains had a higher number of glycoside hydrolases and glycosyltransferases CAZymes than the other two wine yeasts, which points to great flexibility to both degrade or help synthesize complex compounds, which will lead to a release of glycosidically bound flavor compounds (such as terpenes and norisoprenoids) from naturally present grape glycosides and, therefore, most likely have a positive effect on wine mouthfeel and aroma $[48,49]$.

Functional annotation of Clavispora santaluciae was also accomplished using KEGG Mapper-Reconstruct Pathway tool [32,34]. This tool completed KO-based mapping against KEGG databases, allowing us to visualize reconstructed global maps of metabolic pathways. Further, we used Reconstruct Pathway to evaluate pathway completeness for all five strains, together with the type strains of Clavispora fructus, Clavispora lusitaniae, and Saccharomyces cerevisiae. A total of 170 metabolic pathways were analyzed, and the results were categorized in a comparative heatmap (Supplementary Data S3). Reconstructed metabolic pathways of Clavispora santaluciae showed inter-strain differences, mostly in the strain LB-NB-3.3, in comparison with the remaining four, as a reflex of its isolation source. Most evident differences were detected in the citrate cycle, sphingosine degradation, threonine biosynthesis, and glutathione biosynthesis. Type strain $\mathrm{A} 1.18^{\mathrm{T}}$ showed marked differences from the other isolates, as it lacked some genes related to sulfate assimilation-namely, "assimilatory and dissimilatory sulfate reduction" (KEGG modules M00176 and M00596) and "sulfate-sulfur assimilation" (KEGG module M00616). 
Comparison with other strains found in winemaking environments revealed similarity in the completeness of metabolic pathways (Supplementary Data S3), but with some important differences: (a) all Clavispora santaluciae strains lacked half the genes involved in tyrosine biosynthesis, in particular the ones responsible for the conversion of chorismite to tyrosine, as this pathway was complete in the other relevant wine yeast species; (b) KEGG modules M00013 (malonate semialdehyde pathway), M00143 (NADG dehydrogenase), M00066 (lactosylceramide biosynthesis), M00546 (purine degradation), M00133 (polyamine biosynthesis), and M00793 (rhamnose biosynthesis) revealed higher level of completeness in yeasts from Metschnikowiaceae than in S. cerevisiae; (c) module for Leucine degradation revealed higher completeness of metabolic pathways in Clavispora santaluciae, compared with Clavispora lusitaniae.

\subsection{Interspecific Genomic Variability of Metschnikowiaceae}

Comparative genomics between Clavispora santaluciae and the other species of the Metschnikowiaceae was evaluated using the pairwise average nucleotide identity values (ANI in \%), genome size, number of shared genes using type strain $\mathrm{A} 1.18^{\mathrm{T}}$ as query, and percentage of guanine-cytosine bases (GC) in the genome sequences. Figure 3 shows that the number of homologous coding genes is not correlated with the genome size of Metschnikowiaceae. As sequencing of ribosomal DNA regions has shown [4], Clavispora santaluciae is the closest relative to Clavispora fructus and Clavispora lusitaniae, with the ANI similarity of $78.6 \%$ and $73.6 \%$, respectively. Although all the genomes in the present work were reannotated, using the same pipeline in order to avoid incongruences, care must be taken when analyzing genome sizes, since different sequencing technologies and assembly approaches were used by the different authors.

The haploid genome of Clavispora santaluciae is interestingly small (average size of $10.9 \mathrm{Mb}$ ), in contrast to the average $15.3 \mathrm{Mb}$ of the other species of this family (Figure $3 \mathrm{~A}$ ). This small genome size is also reflected in the lower number of protein-coding genes (6049) identified on average for the five Clavispora santaluciae which is similar to the average number (5972) determined in the other Clavispora species but significantly lower than for the other species of Metschnikowiaceae (on average 6744; Figure 3B). An extreme example is Metschnikowia fructicola, with more than 10,000 genes revealed by our genome annotation pipeline, which is a significant increase, compared with previous genome annotation (8629) of this species [46]. From Figure 3 we can observe a large diversity between species of Metschnikowia in terms of their genome size (panel A) and the number of coding genes (panel B), while Clavispora/(Candida) strains generally have smaller genome sizes and fewer predicted proteins. This fact could be related to evolutionary constraints, as species of Metschnikowia are usually associated with diverse environments and substrates, while Clavispora/(Candida) yeasts are typically associated with a few habitats. In particular, Clavispora santaluciae yeasts were only isolated from grapes until now. The smaller genome size and its lower number of coding genes could be related to a lower capability to adapt to new environments. Although a direct link between small genome size and evolutionary plasticity has not yet been established, to our knowledge, some reports link these features in recent years. For example, Steenwyk et al. in 2019 [50] showed that yeasts of the genus Hanseniaspora benefit from their reduced genome sizes by the ability to grow rapidly. They showed that the two Hanseniaspora lineages exhibit very high evolutionary rates and that the lineage had lost many of the genes involved in cell cycle and DNA repair mechanisms during evolution, and has, therefore, been able to diversify more rapidly. On the other hand, other studies show that yeasts with larges genomic sequences have redundant genomes, linked to a strong tendency for map dispersion, visible by duplication of non-coding RNAs, the spread of tRNA genes, and a high number of tRNA genes, as is the case of Yarrowia lipolytica [51]. One last example refers to the evolution of Saccharomycotina yeasts [52], for which it was shown that genes seem to be very rarely gained by horizontal gene transfer, while gene losses are more common, along with the loss of whole sets of genes in some pathways in some species. 


\section{A}

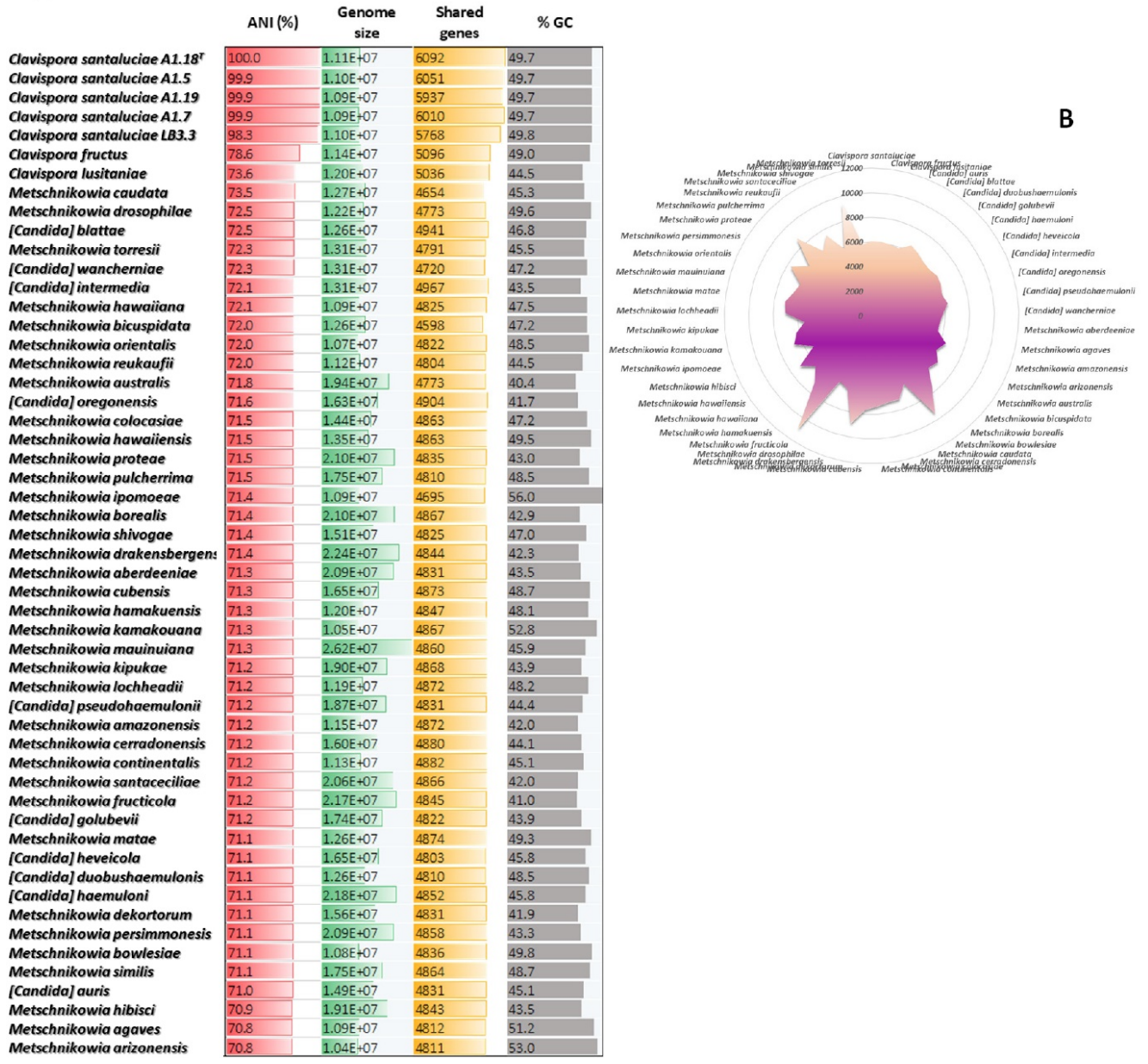

Figure 3. Comparative genomics of Metschnikowiaceae yeasts: (A) average nucleotide identity (\% ANI), genome size ( $\mathrm{Mbp})$, number of coding genes, and percentage of GC among the complete genomes of Metschnikowiaceae species; (B) number of coding genes across the Metschnikowiaceae family.

\subsection{Phylogenomics of Metschnikowiacea}

The phylogenomics of Metschnikowiaceae was determined for all 121 strains (48 species) with complete genomes available, as well as the 5 Clavispora santaluciae whose genomes were sequenced and assembled in the present work. The complete proteome of the type strain A1.18 ${ }^{\mathrm{T}}$ (6092 proteins) was used in a BLASTP analysis against the Metschnikowiaceae proteomic database (with two outgroups), composed in the current study, and a total of 2362 proteins had homologs in the 126 yeasts. The phylogenetic tree highlighted in Figure 4 represents the alignment of the core concatenated proteins. This phylogram represents the most comprehensive phylogenetic assessment of Metschnikowiaceae in which complete genomes were analyzed. As expected, the five Clavispora santaluciae strains formed a homologous clade (highlighted in red in Figure 4), separated from Clavispora fructus, and from strains of Clavispora lusitaniae. The phylogenetic distributions observed with our genome analysis generally agree with the taxonomic phylogeny shown before [4], using alignments of the ITS and D1/D2 regions, with minor exceptions. In the complete-genome analysis, strains $\mathrm{A} 1.18^{\mathrm{T}}$ and $\mathrm{A} 1.19$ were revealed to be closest relatives and separated from strains A1.7 and A1.5. The strain LB-NB-3.3 isolated from Italy was most distantly related. However, the phylogeny of ITS and D1/D2 regions showed the highest similarity between strains $\mathrm{A} 1.18^{\mathrm{T}}$ and A1.5. This separation between later strains, when analyzing complete genomes, can also be observed in the dot plots of Figure 1, and stresses the need for complete-genome analysis to establish robust phylogenies. Species of (Candida) were grouped on a common clade, separated from the "true" Clavispora yeasts (blue box, 
Figure 4), with the exception of (Candida) golubevii and (Candida) wancherniae, which are highlighted by pink boxes in Figure 4.

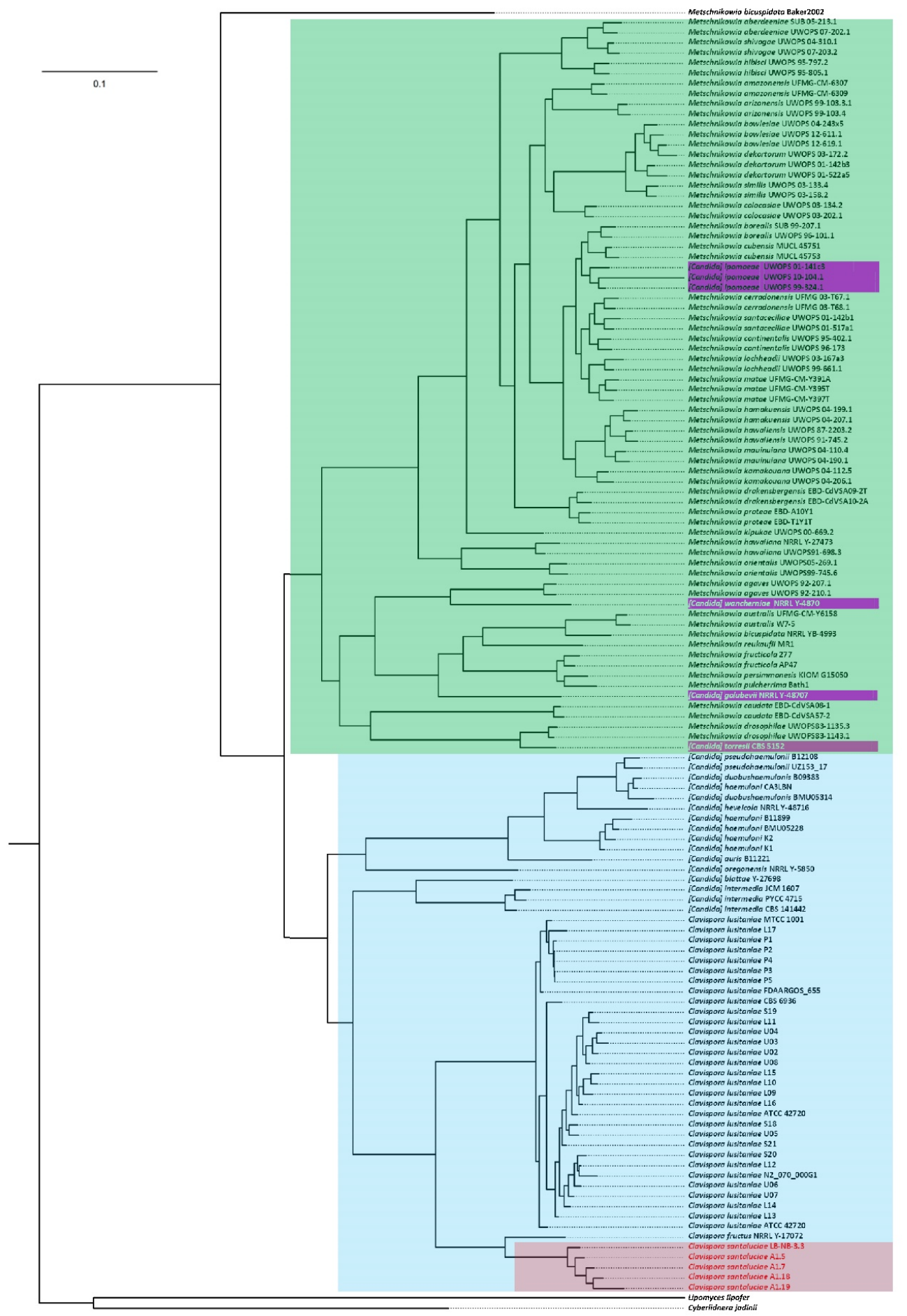

Figure 4. Phylogram of Metschnikowiaceae family showing the core proteome of 126 yeast strains (alignment of 2362 common proteins). Clavispora santaluciae genomes, sequenced in the present work, are highlighted using a red box, while Clavispora/Candida and Metschnikowia genera are highlighted by blue and green boxes, respectively. Incongruent locations are highlighted by purple boxes. Phylogenetic reconstruction was performed by considering maximum likelihood and 500 bootstrap replicates of the concatenated alignments. Bootstrap values were omitted as they were $100 \%$ for all branches. 
Bootstrap values of $100 \%$ were found for all branches, confirming the robustness of the phylogram. Monophyletic branches were obtained for all species with more than one strain, with few exceptions: (a) strain $M$. dekortorum UWOPS 03-172.2 clustered together with the two strains of $M$. bowlesiae, and separately from the other two strains of M. dekortorum; (b) one of the three strains of (Candida) haemuloni-CA3LBN-clustered in a monophyletic branch with (Candida) duobushaemulonis, far from other strains of the same species; (c) strain M. bicuspidata Baker2002 was placed outside the main group of Metschnikowiaceae strains, serving as an outgroup of this larger group and showing clear differences from the strain M. bicuspidata NRRL YB-4993. This last observation needs careful validation because, in the work of Lachance et al. [13], M. bicuspidata NRRL YB-4993 was used as an outgroup in the established phylogeny of Metschnikowia, to root the tree, under the justification of being divergent from the remaining large-spore species. However, in the current work, in addition to the large difference detected, in terms of genomic contents, between both available genomes of $M$. bicuspidata, a similarity was also observed between type strain NRRL YB-4993 and M. australis and M. reukaufii. In addition, in the work of Shen et al. [14], M. bicuspidata was also placed outside the main Metschnikowia species group, clustered with the type strain of Candida golubevii. This fact points to the importance to include different strains in phylogenetic analysis to clarify species positioning. In the future, additional $M$. bicuspidata strains should be sequenced and included in a phylogenomic analysis in order to clarify the position of this species.

The two other incongruent (Candida) inclusions described above as having bootstrap support of $100 \%$ in the phylogram of Figure 4-namely, (Candida) golubevii and (Candida) wancherniae, represent clear candidate species whose nomenclature needs to be revised and included in the Metschnikowia genus. These species had also particular placements in the phylogeny established by Shen et al. [14], presenting an intermediate position between (Candida) and Metschnikowia genera.

\section{Conclusions}

Clavispora santaluciae is a novel non-Sacharomyces yeast species, recently isolated from grapes of Azores vineyards, a Portuguese archipelago with particular environmental conditions, and from Italian grapes infected with Drosophila suzukii. In the current work, complete genomes of all the described Clavispora santaluciae strains were sequenced, assembled, and annotated. With this work, we increase the number of Metschnikowiaceae yeasts with the sequenced and annotated genome. By using a combination of long- and short-read sequencing technologies to sequence strains' genomes, we were able to obtain high-quality and complete DNA sequences, which allowed us to predict a high number of coding sequences and robust sequencing statistics. This high number of protein-coding genes might not be related to any particularity of this yeast's genome but rather a consequence of an improvement of the sequencing technology and the annotation pipeline.

Genome comparison revealed particular differences between strains of Clavispora santaluciae, reflecting their isolation from two different ecological niches-Azorean and Italian vineyards - as well as mechanisms of adaptation to the intricate and arduous environment features of the geographical location from which they were isolated. In particular, the differences in terms of number of coding genes (shared and unique), number of the transposable elements, the amount and diversity of non-coding RNAs, and enzymatic potential of each strain through CAZyome analysis were detailed in the present work to unravel mechanisms of adaptation to both environments. These differences, primarily the ones found between Italian and Portuguese strains, echoes mainly climatic differences in the strains ' origin. While Italian grapes were obtained in vineyards from the variety Vernatsch, for which the influential climatic condition is the warm, moist, and continental weather, grapes from Azorean vineyards are subject to a particular and aggressive microclimate due to the basaltic stone soils. Results show different adaptation mechanisms underlying the occurrence of these yeasts in nature, as, for example, the absence of transposable elements 
in the strain isolated from Italy, which was sufficient to leave a marked fingerprint in their genomes.

A future increase in the number of Clavispora santaluciae strains will allow the use of populational analysis methods to unravel new mechanisms of adaptation to the environment and to explore new practical applications of these isolates [53-56]. In detail, genome-wide environmental associations could be explored recurring to algorithms described for targeted mapping [57], linkage disequilibrium (LD) measures could allow the dissection of genetic diversity [58], and new approaches based on machine learning algorithms can open new doors to discover novel biotechnological applications [59,60].

Comparison of Clavispora santaluciae with other yeast species successfully unraveled the presence of distinct traits that elevate this species potential for biotechnological applications. The small genome size combines a high number of protein-coding genes and a high percentage of metabolic pathways completeness. In its CAZyome, Clavispora santaluciae revealed a high number of glycoside hydrolases and glycosyltransferases, even higher than the ones existing in traditionally used wine yeasts. This discovery reflects great flexibility to both degrade or synthesize complex compounds, both with potential interest in winemaking and in other biotechnological industries.

Using complete genomes of Metschnikowiaceae, we presented the largest ever phylogenetic assessment of this yeast family, highlighting particular differences to other phylograms with less robustness that use only some parts of the ribosomal genes. With this analysis, it was possible to identify three (Candida) species whose nomenclature needs to be revised. The growing knowledge about this yeast family unravels new potential applications of these species as the high genomic plasticity may also correlate to a larger phenotypic diversity and a higher propensity to adapt to new environments.

Supplementary Materials: The following are available online at https:/ /www.mdpi.com/article/ 10.3390/jof8010052/s1. Supplementary Data S1: Clavispora santaluciae genome annotations, Supplementary Data S2: non-coding RNAs predicted in the genome of Clavispora santaluciae, Supplementary Data S3: Heatmap representing pathway completeness in Clavispora santaluciae, Clavispora lusitaniae, Clavispora fructus, and Saccharomyces cerevisiae. Completeness values were obtained using KEGG Mapper-Reconstruct tool, and organized using KEGG Modules information.

Author Contributions: Conceptualization, P.S., N.Č., Y.R.D., C.P. and R.F.-D.; methodology, R.F.-D., N.Č., T.R. and P.S.; software, R.F.-D., N.Č., T.R. and P.S.; validation, R.F.-D., N.Č., T.R., C.P., M.J.S. and P.S.; writing —original draft preparation, R.F.-D., N.C.., T.R. and P.S.; writing-review and editing, R.F.-D., N.Č., T.R., Y.R.D., J.D.-N., C.P., M.J.S. and P.S. All authors have read and agreed to the published version of the manuscript.

Funding: This work was supported by the UID/BIA/04050/2013 (POCI-01-0145-FEDER-007569) and UID/BIA/04050/2019 strategic programs, by the project PTDC/BIA-MIC/32059/2017 (TODOMICS: Omics approaches towards the industrial exploitation of Torulaspora delbrueckii: Elucidation of the molecular basis underlying complex cellular traits), which is funded by national funds through the Fundação para a Ciência e Tecnologia (FCT), by the Slovenian Research Agency (P4-0116, and MRICUL ZIM, IP-0510 to N.Č)", and by the European Regional Development Fund (ERDF) through the COMPETE2020 Programa Operacional Competitividade e Internacionalização (POCI) and Sistema de Apoio à Investigação Científica e Tecnológica (SAICT).

Institutional Review Board Statement: Not applicable.

Informed Consent Statement: Not applicable.

Data Availability Statement: The sequencing data are available at NCBI BioProject ID PRJNA784374.

Conflicts of Interest: The authors declare no conflict of interest. 


\section{References}

1. Drumonde-Neves, J.; Franco-Duarte, R.; Lima, T.; Schuller, D.; Pais, C. Association between grape yeast communities and the vineyard ecosystems. PLoS ONE 2017, 12, e0169883. [CrossRef] [PubMed]

2. Drumonde-Neves, J.; Franco-Duarte, R.; Lima, T.; Schuller, D.; Pais, C. Yeast biodiversity in vineyard environments is increased by human intervention. PLoS ONE 2016, 11, e0160579. [CrossRef]

3. Drumonde-Neves, J.; Franco-Duarte, R.; Vieira, E.; Mendes, I.; Lima, T.; Schuller, D.; Pais, C. Differentiation of Saccharomyces cerevisiae populations from vineyards of the Azores Archipelago: Geography vs. Ecology. Food Microbiol. 2018, 74, 151-162. [CrossRef] [PubMed]

4. $\quad$ Drumonde-Neves, J.; Čadež, N.; Domínguez, Y.R.; Gallmetzer, A.; Schuller\#, D.; Lima, T.; Pais, C.; Franco-Duarte, R. Clavispora santaluciae f.a., sp. nov., a novel ascomycetous yeast species isolated from grapes. Int. J. Syst. Evol. Microbiol. 2020, 70, 6307-6312 [CrossRef]

5. Gárdonyi, M.A.; Österberg, M.A.; Rodrigues, C.; Spencer-Martins, I.; Hahn-Hägerdal, B. High capacity xylose transport in Candida intermedia PYCC 4715. FEMS Yeast Res. 2003, 3, 45-52. [CrossRef]

6. Geijer, C.; Faria-Oliveira, F.; Moreno, A.D.; Stenberg, S.; Mazurkewich, S.; Olsson, L. Genomic and transcriptomic analysis of Candida intermedia reveals the genetic determinants for its xylose-converting capacity. Biotechnol. Biofuels 2020, 13, 48. [CrossRef] [PubMed]

7. Moreno, A.D.; Tomás-Pejó, E.; Olsson, L.; Geijer, C. Candida intermedia CBS 141442: A novel glucose/xylose co-fermenting isolate for lignocellulosic bioethanol production. Energies 2020, 13, 5363. [CrossRef]

8. Drumonde-Neves, J.; Fernandes, T.; Lima, T.; Pais, C.; Franco-Duarte, R. Learning from 80 years of studies: A comprehensive catalogue of non-Saccharomyces yeasts associated with viticulture and winemaking. FEMS Yeast Res. 2021, 21, foab017. [CrossRef]

9. Mingorance-Cazorla, L.; Clemente-Jiménez, J.M.; Martínez-Rodríguez, S.; Las Heras-Vázquez, F.J.; Rodríguez-Vico, F. Contribution of different natural yeasts to the aroma of two alcoholic beverages. World J. Microbiol. Biotechnol. 2003, 19, 297-304. [CrossRef]

10. Morata, A.; Loira, I.; Escott, C.; del Fresno, J.M.; Bañuelos, M.A.; Suárez-Lepe, J.A. Applications of Metschnikowia pulcherrima in wine biotechnology. Fermentation 2019, 5, 63. [CrossRef]

11. Daniel, H.M.; Lachance, M.A.; Kurtzman, C.P. On the reclassification of species assigned to Candida and other anamorphic ascomycetous yeast genera based on phylogenetic circumscription. Antonie Leeuwenhoek 2014, 106, 67-84. [CrossRef] [PubMed]

12. Kurtzman, C.P.; Robnett, C.J.; Basehoar, E.; Ward, T.J. Four new species of Metschnikowia and the transfer of seven Candida species to Metschnikowia and Clavispora as new combinations. Antonie Leeuwenhoek 2018, 111, 2017-2035. [CrossRef] [PubMed]

13. Lachance, M.-A.; Hurtado, E.; Hsiang, T. A stable phylogeny of the large-spored Metschnikowia clade. Yeast 2016, 33, 261-275 [CrossRef] [PubMed]

14. Shen, X.X.; Opulente, D.A.; Kominek, J.; Zhou, X.; Steenwyk, J.L.; Buh, K.V.; Haase, M.A.B.; Wisecaver, J.H.; Wang, M.; Doering, D.T.; et al. Tempo and Mode of Genome Evolution in the Budding Yeast Subphylum. Cell 2018, 175, 1533-1545.e20. [CrossRef]

15. Schwartz, K.; Sherlock, G. Preparation of yeast DNA sequencing libraries. Cold Spring Harb. Protoc. 2016, 2016, 871-876. [CrossRef]

16. Koren, S.; Walenz, B.P.; Berlin, K.; Miller, J.R.; Bergman, N.H.; Phillippy, A.M. Canu: Scalable and accurate long-read assembly via adaptive K-mer weighting and repeat separation. Genome Res. 2017, 27, 722-736. [CrossRef] [PubMed]

17. Zimin, A.V.; Marçais, G.; Puiu, D.; Roberts, M.; Salzberg, S.L.; Yorke, J.A. The MaSuRCA genome assembler. Bioinformatics 2013, 29, 2669-2677. [CrossRef] [PubMed]

18. Zimin, A.V.; Salzberg, S.L. The genome polishing tool POLCA makes fast and accurate corrections in genome assemblies. PLoS Comput. Biol. 2020, 16, e1007981. [CrossRef] [PubMed]

19. Alonge, M.; Soyk, S.; Ramakrishnan, S.; Wang, X.; Goodwin, S.; Sedlazeck, F.J.; Lippman, Z.B.; Schatz, M.C. RaGOO: Fast and accurate reference-guided scaffolding of draft genomes. Genome Biol. 2019, 20, 224. [CrossRef]

20. Mikheenko, A.; Prjibelski, A.; Saveliev, V.; Antipov, D.; Gurevich, A. Versatile genome assembly evaluation with QUAST-LG. Bioinformatics 2018, 34, i142-i150. [CrossRef]

21. Weib, C.L.; Pais, M.; Cano, L.M.; Kamoun, S.; Burbano, H.A. nQuire: A statistical framework for ploidy estimation using next generation sequencing. BMC Bioinform. 2018, 19, 122. [CrossRef]

22. Seppey, M.; Manni, M.; Zdobnov, E. BUSCO: Assessing Genome Assembly and Annotation Completeness. In Methods in Molecular Biology; Humana: New York, NY, USA, 2019; Volume 1962, pp. 227-245. ISBN 9781493991730.

23. Yoon, S.-H.; Ha, S.-M.; Lim, J.; Kwon, S.; Chun, J. A large-scale evaluation of algorithms to calculate average nucleotide identity. Antonie Leeuwenhoek 2017, 110, 1281-1286. [CrossRef] [PubMed]

24. Stanke, M.; Schöffmann, O.; Morgenstern, B.; Waack, S. Gene prediction in eukaryotes with a generalized hidden Markov model that uses hints from external sources. BMC Bioinform. 2006, 7, 62. [CrossRef] [PubMed]

25. Stanke, M.; Morgenstern, B. AUGUSTUS: A web server for gene prediction in eukaryotes that allows user-defined constraints Nucleic Acids Res. 2005, 33, W465-W467. [CrossRef]

26. Cui, X.; Lu, Z.; Wang, S.; Jing-Yan Wang, J.; Gao, X. CMsearch: Simultaneous exploration of protein sequence space and structure space improves not only protein homology detection but also protein structure prediction. Bioinformatics 2016, 32, i332-i340. [CrossRef] 
27. Arias-Carrasco, R.; Vásquez-Morán, Y.; Nakaya, H.I.; Maracaja-Coutinho, V. StructRNAfinder: An automated pipeline and web server for RNA families prediction. BMC Bioinform. 2018, 19, 55. [CrossRef] [PubMed]

28. Griffiths-Jones, S.; Moxon, S.; Marshall, M.; Khanna, A.; Eddy, S.R.; Bateman, A. Rfam: Annotating non-coding RNAs in complete genomes. Nucleic Acids Res. 2005, 33, D121-D124. [CrossRef]

29. Cantalapiedra, C.P.; Hernández-Plaza, A.; Letunic, I.; Bork, P.; Huerta-Cepas, J. eggNOG-mapper v2: Functional Annotation, Orthology Assignments, and Domain Prediction at the Metagenomic Scale. Mol. Biol. Evol. 2021, 38, 5825-5829. [CrossRef]

30. Tatusov, R.L.; Galperin, M.Y.; Natale, D.A.; Koonin, E.V. The COG database: A tool for genome-scale analysis of protein functions and evolution. Nucleic Acids Res. 2000, 28, 33-36. [CrossRef]

31. Kanehisa, M. KEGG: Kyoto Encyclopedia of Genes and Genomes. Nucleic Acids Res. 2000, 28, 27-30. [CrossRef] [PubMed]

32. Kanehisa, M.; Sato, Y.; Kawashima, M. KEGG mapping tools for uncovering hidden features in biological data. Protein Sci. 2021. [CrossRef] [PubMed]

33. Cantarel, B.I.; Coutinho, P.M.; Rancurel, C.; Bernard, T.; Lombard, V.; Henrissat, B. The Carbohydrate-Active EnZymes database (CAZy): An expert resource for glycogenomics. Nucleic Acids Res. 2009, 37, D233-D238. [CrossRef] [PubMed]

34. Kanehisa, M.; Sato, Y. KEGG Mapper for inferring cellular functions from protein sequences. Protein Sci. 2020, $29,28-35$. [CrossRef] [PubMed]

35. Santiago, C.; Rito, T.; Vieira, D.; Fernandes, T.; Pais, C.; Sousa, M.J.; Soares, P.; Franco-Duarte, R. Improvement of torulaspora delbrueckii genome annotation: Towards the exploitation of genomic features of a biotechnologically relevant yeast. J. Fungi 2021, 7, 287. [CrossRef]

36. Sun, Y.B. FasParser: A package for manipulating sequence data. Zool. Res. 2017, 38, 110-112. [CrossRef]

37. Nguyen, L.T.; Schmidt, H.A.; Von Haeseler, A.; Minh, B.Q. IQ-TREE: A fast and effective stochastic algorithm for estimating maximum-likelihood phylogenies. Mol. Biol. Evol. 2015, 32, 268-274. [CrossRef]

38. Durrens, P.; Klopp, C.; Biteau, N.; Fitton-Ouhabi, V.; Dementhon, K.; Accoceberry, I.; Sherman, D.J.; Noël, T. Genome Sequence of the Yeast Clavispora lusitaniae Type Strain CBS 6936. Genome Announc. 2017, 5, 30-31. [CrossRef]

39. Kannan, A.; Asner, S.A.; Trachsel, E.; Kelly, S.; Parker, J.; Sanglard, D. Comparative Genomics for the Elucidation of Multidrug Resistance in Candida lusitaniae. MBio 2019, 10, e02512-19. [CrossRef]

40. Moreno, A.D.; Tellgren-Roth, C.; Soler, L.; Dainat, J.; Olsson, L.; Geijer, C. Complete Genome Sequences of the Xylose-Fermenting Candida intermedia Strains CBS 141442 and PYCC 4715. Genome Announc. 2017, 5, e00138-17. [CrossRef]

41. Garbarino, J.E.; Gibbons, I.R. Expression and genomic analysis of midasin, a novel and highly conserved AAA protein distantly related to dynein. BMC Genom. 2002, 3, 18. [CrossRef]

42. Franco-Duarte, R.; Bigey, F.; Carreto, L.; Mendes, I.; Dequin, S.; Santos, M.A.S.; Pais, C.; Schuller, D. Intrastrain genomic and phenotypic variability of the commercial Saccharomyces cerevisiae strain Zymaflore VL1 reveals microevolutionary adaptation to vineyard environments. FEMS Yeast Res. 2015, 15, fov063. [CrossRef] [PubMed]

43. Franco-Duarte, R.; Umek, L.; Zupan, B.; Schuller, D. Computational approaches for the genetic and phenotypic characterization of a Saccharomyces cerevisiae wine yeast collection. Yeast 2009, 26, 675-692. [CrossRef]

44. Franco-Duarte, R.; Umek, L.; Mendes, I.; Castro, C.C.; Fonseca, N.; Martins, R.; Silva-Ferreira, A.C.; Sampaio, P.; Pais, C.; Schuller, D. New integrative computational approaches unveil the Saccharomyces cerevisiae pheno-metabolomic fermentative profile and allow strain selection for winemaking. Food Chem. 2016, 211, 509-520. [CrossRef]

45. Davies, G.J.; Gloster, T.M.; Henrissat, B. Recent structural insights into the expanding world of carbohydrate-active enzymes. Curr. Opin. Struct. Biol. 2005, 15, 637-645. [CrossRef]

46. Piombo, E.; Sela, N.; Wisniewski, M.; Hoffmann, M.; Gullino, M.L.; Allard, M.W.; Levin, E.; Spadaro, D.; Droby, S. Genome sequence, assembly and characterization of two Metschnikowia fructicola strains used as biocontrol agents of postharvest diseases. Front. Microbiol. 2018, 9, 593. [CrossRef]

47. Fernandes, T.; Silva-Sousa, F.; Pereira, F.; Rito, T.; Soares, P.; Franco-Duarte, R.; Sousa, M.J. Biotechnological Importance of Torulaspora delbrueckii: From the Obscurity to the Spotlight. J. Fungi 2021, 7, 712. [CrossRef]

48. Sahay, S. Wine enzymes: Potential and practices. Enzym. Food Biotechnol. Prod. Appl. Futur. Prospect. 2018, 73-92. [CrossRef]

49. Daenen, L.; Saison, D.; Sterckx, F.; Delvaux, F.R.; Verachtert, H.; Derdelinckx, G. Screening and evaluation of the glucoside hydrolase activity in Saccharomyces and Brettanomyces brewing yeasts. J. Appl. Microbiol. 2008, 104, 478-488. [CrossRef] [PubMed]

50. Steenwyk, J.L.; Opulente, D.A.; Kominek, J.; Shen, X.X.; Zhou, X.; Labella, A.L.; Bradley, N.P.; Eichman, B.F.; Čadež, N.; Libkind, D.; et al. Extensive loss of cell-cycle and DNA repair genes in an ancient lineage of bipolar budding yeasts. PLoS Biol. 2019, 17, e3000255. [CrossRef] [PubMed]

51. Dujon, B.; Sherman, D.; Fischer, G.; Durrens, P.; Casaregola, S.; Lafontaine, I.; de Montigny, J.; Marck, C.; Neuvéglise, C.; Talla, E.; et al. Genome evolution in yeasts. Nature 2004, 430, 35-44. [CrossRef] [PubMed]

52. Scannell, D.R.; Butler, G.; Wolfe, K.H. Yeast genome evolution-the origin of the species. Yeast 2007, 24, 929-942. [CrossRef]

53. Gómez, S.; Berdugo, S.; Mena, R. Occurrence of indigenous arbuscular mycorrhizal fungi associated with the rhizosphere of the naidí palm in Colombia. Cienc. Tecnol. Agropecu. 2020, 21, e1275.

54. Restrepo-Correa, S.; Pineda-Meneses, E.; Rios-Osorio, L. Mechanisms of action of fungi AND bacteria used as biofertilizers in agricultural soils: A systematic review. Corpoica. Tecnol. Agropecu. 2017, 18, 335-351. 
55. Youdkes, D.; Helman, Y.; Burdman, S.; Matan, O.; Jurkevitch, E. Potential control of potato soft rot disease by the obligate predators bdellovibrio and like organisms. Appl. Environ. Microbiol. 2020, 86, e02543-19. [CrossRef] [PubMed]

56. Leon-Ttacca, B.; Arévalo-Gardini, E.; Bouchon, A.S. Sudden death of Theobroma cacao L. caused by Verticillium dahliae Kleb. In Peru and its in vitro biocontrol. Cienc. Tecnol. Agropecu. 2019, 20, 133-148. [CrossRef]

57. López-Hernández, F.; Cortés, A.J. Last-Generation Genome-Environment Associations Reveal the Genetic Basis of Heat Tolerance in Common Bean (Phaseolus vulgaris L.). Front. Genet. 2019, 10, 954. [CrossRef] [PubMed]

58. Blair, M.W.; Cortés, A.J.; Farmer, A.D.; Huang, W.; Ambachew, D.; Varma Penmetsa, R.; Carrasquilla-Garcia, N.; Assefa, T.; Cannon, S.B. Uneven recombination rate and linkage disequilibrium across a reference SNP map for common bean (Phaseolus vulgaris L.). PLoS ONE 2018, 13, e0189597. [CrossRef] [PubMed]

59. Cortés, A.J.; López-Hernández, F.; Osorio-Rodriguez, D. Predicting Thermal Adaptation by Looking Into Populations' Genomic Past. Front. Genet. 2020, 11, 564515. [CrossRef]

60. Cortés, A.J.; López-Hernández, F. Harnessing crop wild diversity for climate change adaptation. Genes 2021, 12, 783. [CrossRef] 